\title{
Correspondence
}

Journal of Medical Genetics, 1981, 18, 399-400

\section{Hereditary motor and sensory neuropathy}

SIR,

The report in the October issue of Journal of Medical Genetics by Harding and Thomas ${ }^{1}$ has added important new information to our understanding of the genetic heterogeneity of the hereditary motor and sensory neuropathies (HMSN). However, I believe the authors arrived at a premature conclusion regarding the genetic counselling of families with type I HMSN. Based on the fact that reduced nerve conduction velocities may sometimes be found in infants at risk, they state "a clinically and electrophysiologically normal subject can therefore be given a zero risk". Although this statement may eventually prove to be correct, all the pertinent information is not yet available. It is true that this condition can sometimes be documented at an early age, and we have found slow motor nerve conduction velocities in a 14-month-old boy whose mother was also affected. Nevertheless, Dr G Kraft and I found that only $14.3 \%$ ( 2 of 14 ) of children at risk in the first decade of life demonstrated definite signs (including nerve conduction velocity slowing) of classic autosomal dominant Charcot-Marie-Tooth neuropathy (type I HMSN). ${ }^{2}$ This was significantly less than the expected $50 \%$. This was a small sample and larger numbers of young children at risk should be studied longitudinally. It is possible that some subjects who have inherited the gene will not show detectable delay in motor nerve conduction velocities until after the first decade of life. The frequency of these possible false negative diagnoses should be determined before such persons are given a 'zero risk'.

Thomas D BIRD

Neurology Section, VA Medical Center, 4435 Beacon Avenue South, Seattle, Washington 98108, USA.

\section{References}

1 Harding AE, Thomas PK. Genetic aspects of hereditary motor and sensory neuropathy (types I and II). $J$ Med Genet 1980;17:329-36.

2 Bird TD, Kraft GH. Charcot-Marie-Tooth disease: data for genetic counselling relating age to risk. Clin Genet 1978;14:43-9.

This letter was shown to Dr Harding and Professor Thomas who reply as follows.
SiR,

Dr Bird has removed our statement about the 'zero risk' from its context. This referred to "asymptomatic sibs or children of affected subjects from dominantly inherited families (with type I HMSN) who wish to know the risks of producing affected children". At the time of definitive genetic counselling it is likely that such subjects will be of child-bearing age. The question as to whether or not nerve conduction velocity is reduced in the first decade of life in those affected is therefore irrelevant. In a very large series of cases, $w^{1}$ have never observed transmission of the disorder by a subject with normal nerve conduction. Drs Bird and $\mathrm{Kraft}^{2}$ found that the proportions of affected children at risk in the 10 to 15 and 10 to 20-year age groups were 50 and $52.4 \%$ respectively (excluding probands) which would support our statement. They do not mention how many children aged 10 or less had had nerve conduction studies performed. It is probable that slowing of nerve conduction velocity precedes abnormal clinical signs. It is also not absolutely clear whether Bird and Kraft excluded cases of type II HMSN from their study. Such cases may have "unequivocally slow motor nerve conduction velocities".

The exact age at which nerve conduction becomes abnormal is likely to remain uncertain in view of the ethical and technical problems involved in investigating apparently normal young infants. All that can be said is that the findings of Dyck and colleagues, ${ }^{3}$ and examples such as the 14-month-old boy mentioned by Dr Bird, indicate that demyelination may be present from very early childhood.

\section{A E Harding and P K Thomas Department of Neurological Science, The Royal Free Hospital, Pond Street, London NW3 $2 Q G$.}

\section{References}

1 Harding AE, Thomas PK. The clinical features of hereditary motor and sensory neuropathy types I and II. Brain 1980;103:259-80.

2 Bird TD, Kraft GH. Charcot-Marie-Tooth disease: data for genetic counselling relating age to risk. Clin Genet 1978;14:43-9.

3 Dyck PJ, Lambert EH, Mulder DW. Charcot-MarieTooth disease: nerve conduction and clinical studies of a large kinship. Neurology (Minneap) 1963;13:1-11. 